\title{
MULTIPLEXED PROTEOMICS USING TWO ORDERS OF MAGNITUDE ENHANCED DIELECTROPHOREIS: A COMPREHENSIVE ELECTRICAL AND ELECTROTHERMAL DESIGN METHODOLOGY
}

Sam Emaminejad ${ }^{1,2,3}$, Michael T. Barako ${ }^{4}$, Ronald W. Davis ${ }^{2,3}$, Robert W. Dutton ${ }^{1}$, Kenneth E. Goodson ${ }^{4}$, and Mehdi Javanmard ${ }^{1,2,3}$

${ }^{1}$ Electrical Engineering Department, Stanford University, Stanford, CA, USA

${ }^{2}$ Stanford School of Medicine, Stanford, CA, USA

${ }^{3}$ Stanford Genome Technology Center, Stanford University, Palo Alto, CA, USA

${ }^{4}$ Mechanical Engineering Department, Stanford University, Stanford, CA, USA

\begin{abstract}
We present a methodological approach to analyze an enhanced dielectrophoresis (DEP) system from both a circuit analysis and an electrothermal view points. In our developed model, we have taken into account various phenomena and constraints such as voltage degradation (due to the presence of the protecting oxide layer), oxide breakdown, instrumentation limitations, and thermal effects. The results from this analysis are applicable generally to a wide variety of geometries and high voltage microsystems. Here, these design guidelines were applied to develop a robust electronic actuation system to perform a multiplexed bead-based protein assay. For proof of concept, we illustrated 16-plex actuation capability of our device to elute micron-sized beads that are bound to the surface through anti-IgG and IgG interaction which is on the same order of magnitude in strength as typical antibody-antigen interactions. In addition to its application in multiplexed protein analysis, our platform can be potentially utilized to statistically characterize the strength profile of biological bonds, since the multiplexed format allows for high throughput force spectroscopy using the array of uDEP devices, under the same buffer and assay preparation conditions.
\end{abstract}

\section{INTRODUCTION}

Using enhanced negative dielectrophoresis (nDEP), or ultra-DEP (uDEP), we demonstrate an electronic actuation system to perform a multiplexed bead-based protein assay (Figure 1). We pattern an array of proteins along a single microfluidic channel, where each element targets a specific secondary protein coated on micron-sized beads in the subsequently introduced sample. Below each element of the array, we have a pair of addressable interdigitated electrodes (IDE). By selectively applying voltage at the terminals of each IDE pair, the DEP force detaches proteinbound beads from each element of the array, one by one, without disturbing the bound beads in the neighboring regions. The detached beads can be quantified optically or electrically downstream. Here, we illustrate 16-plex actuation capability of our device to elute beads surface-bound through anti-IgG and $\mathrm{IgG}$ interaction which is on the same order of magnitude in strength as typical antibody-antigen interactions.

Previously, using nDEP force in conjunction with shear force and eluting agent, we demonstrated switch-like functionality to elute specifically bound beads from the surface [1]. The eluting agent was used to sufficiently weaken the bindings such that the inherently weak nDEP force would be able to push the bound beads off the surface. Generally, our ability to enhance DEP forces was limited by electrode corrosion when applying voltages beyond $10 \mathrm{~V}$. We enhanced the DEP force by two orders of magnitude by fabricating high voltage tolerant electrodes, where we deposited a pinhole free nanometer-scale thin film oxide as a protective layer, using atomic layer deposition [2,3]. However, this approach imposed a number of challenges that needed to be resolved. From electrical modeling standpoint, deposition of oxide at the electrode-electrolyte interface and the resultant capacitance forms a voltage divider that causes an undesired voltage drop across the thin film, leading to degradation of the electric field (across the solution) and DEP force, and possibly, the breakdown of the oxide. Furthermore, in our high voltage system, the temperature in our channel may rise to the point that electrothermal effects may become dominant and outweigh the DEP effect; preventing DEP to act as a robust localized actuator.

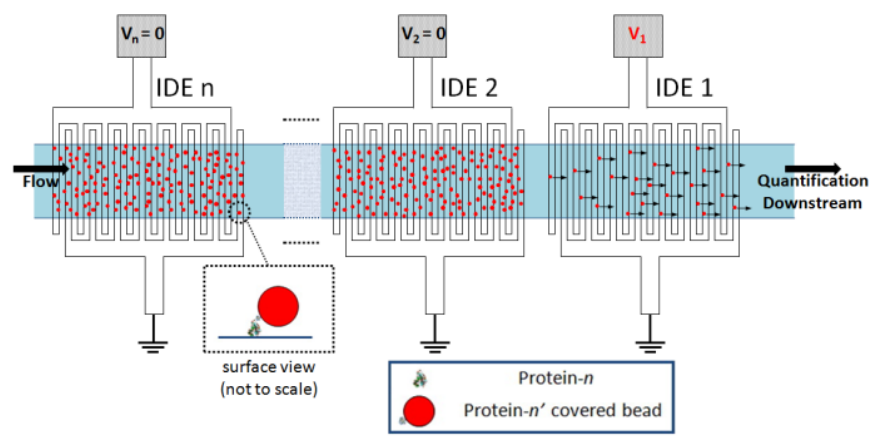

Figure 1: Bead-based multiplexed assay. Each element of array in the capture region is functionalized with a different protein, each targeting a specific protein that is coated on the micron-sized beads. Specifically bound beads on each element of the array are eluted selectively from the array and are quantified downstream (one element at a time). Here, applying voltage $V_{1}$ produces $u D E P$ force, which in turn detaches the specifically bound beads from the surface of the 1st interdigitated electrode pair (i.e. IDE 1). With no voltage applied at the other IDEs, the protein-bound beads on the respective elements remain attached to the surface.

\section{DESIGN METHODOLOGY}

Following the nanometer-scale thin film oxide deposition, our approach focuses on maximizing the DEP force through maximizing the voltage that can be applied across the solution resistance, while meeting all the electrical and thermal constraints. From electrical modeling standpoint, our constraints are oxide breakdown and excitation circuitry's limitation in providing the high levels of voltage and current (required to establish the desired voltage level across the solution resistance). These constraints set an upper-bound on the maximum voltage that can be applied across the solution resistance i.e. $V_{R, \max }$ as captured respectively by the equation below:

$V_{R, \text { max }}=\min \left\{2 E_{B R, o x} \pi \varepsilon_{0} \varepsilon_{o x} s_{e l}\left(\frac{f}{\sigma}\right), \frac{v_{a c, \max }}{\sqrt{1+\left[\left(\frac{1}{\pi \varepsilon_{0} \varepsilon_{o x} s_{e l}}\right)\left(\frac{\sigma}{f}\right) t_{o x}\right]^{2}}}, I_{a c, \max }\left(\frac{s_{e l}}{n \sigma A_{e l}}\right)\right\}$
Solid-State Sensors, Actuators and Microsystems Workshop Hilton Head Island, South Carolina, June 8-12, 2014 
In this equation $E_{B R, o x}$ and $\varepsilon_{o x}$ refer to electric field oxide breakdown and dielectric constant of our ALD thin film, $s_{e l}$ is the spacing between two electrodes, $f$ is the frequency of excitation, $\sigma$ is the solution conductivity, $A_{e l}$ is the area bound by the transverse width of the channel and width of a single electrode, and $n$ is the number of electrode pairs in our interdigitated configuration. $V_{a c, \max }$ and $I_{a c, \max }$ refer to maximum voltage and current amplitude that can be delivered by the signal generator. To better visualize the design space, in Figures 2(a) we illustrated $V_{R, \max }$ for $t_{o x}=10$ $\mathrm{nm}$ while meeting the electrical constraints.

From thermal modeling standpoint, our main constraint is bubble formation due to the increase in the temperature upon applying higher voltages [4]. Assuming bubble formation takes place upon temperature increase of $\Delta T_{\max }, V_{R, \max }$ constrained by thermal limitations can be derived as:

$$
V_{R, \max , t h} \cong \sqrt{\beta\left(\frac{k_{s u b}}{\sigma}\right) \Delta T_{\max }}
$$

In this equation $k_{s u b}$ refers to the thermal conductivity of the substrate (on which electrodes are patterned) and $\beta=2\left(\frac{s_{e l}{ }^{2}}{t_{s u b} w_{e l}}\right)$ captures the role of geometrical dimensions (note in the expression for $\beta, w_{e l}$ refers to the width of the electrodes). For more precise analysis we can calibrate the value of $\beta$ through simulation (Figure 2(b)).

To complete our design methodology, we need to take into account the effect of clausius mossotti factor $f_{C M}$ (which is frequency- and conductivity-dependent) on DEP. As a result, in our design space to achieve maximum DEP force we need to operate in the region where $\operatorname{Re}\left\{f_{C M}\right\} \times V_{R, \max }^{2}$ is maximized, as illustrated in Figure 3.

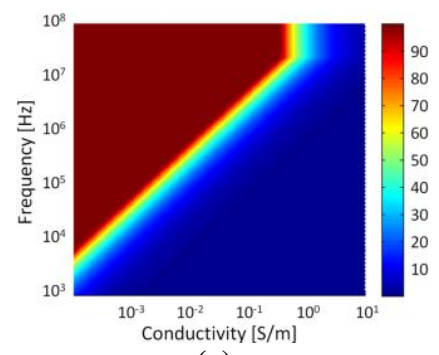

(a)

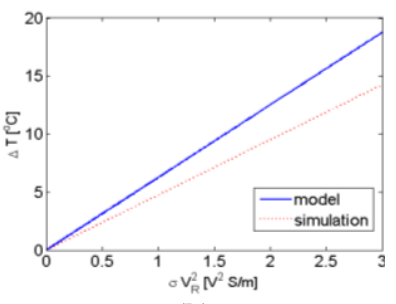

(b)
Figure 2: (a) Representative 'electrical' design space illustrating $V_{\text {solution }}$ for $t_{o x}=10 \mathrm{~nm}$, while meeting the oxide breakdown criterion $\left(E_{B R, o x}=1 \mathrm{~V} / \mathrm{nm}\right)$ and instrumentation limitation (here assumed $V_{a c, \max }=100 \mathrm{~V}, I_{a c, \max }=0.2 \mathrm{~A}$ ) (b) Proportionality of the raise in temperature with the generated power density (setting an upper-bound on $\sigma V^{2}$ solution ). Through calibration with simulation (in our case by $\sim 30 \%$ ) we can precisely model the 'thermal' behavior of the coplanar configuration.

\section{RESULTS AND DISCUSSION}

We confirmed our developed model for electrical interface through impedance spectroscopy (similar to our approach in [2]). Also, through infrared microscopy characterization (Figure 4) we confirmed that DEP force stays dominant in the context of our high voltage microsystem and that electrothermal effects can be neglected.

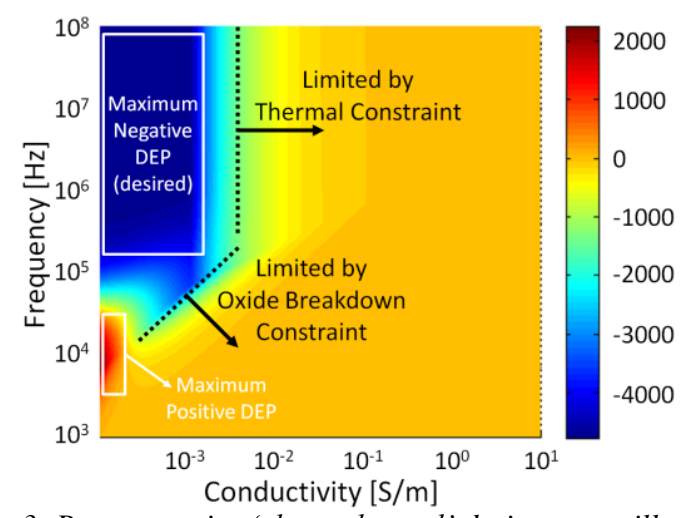

Figure 3: Representative 'electrothermal' design space illustrating relative maximum DEP force (proportional to $\operatorname{Re}\left\{f_{C M}\right\} \times V_{R, \max }{ }^{2}$ ) for a 10-nm deposited oxide that can be achieved for $7 \mu \mathrm{m}$ wide and 7 $\mu m$ spaced IDE pair geometry while meeting the design constraints. The limitations imposed by the dominant design constraints are annotated on the graph. -/+ values correspond to negative/positive DEP. For our application we prefer the most negative value.
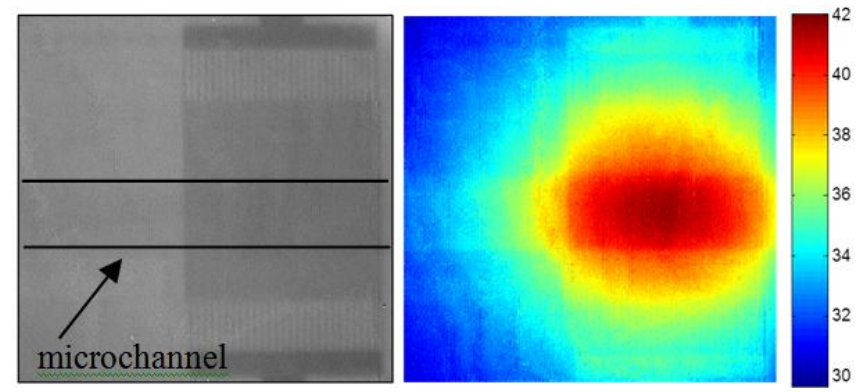

Figure 4: Representative infrared microscopy image of the device, providing a non-invasive measurement of the two-dimensional temperature map of the surface of the wafer. Here, the microchannel is filled with diluted phosphate-buffered saline and $50 V_{p p}$ is applied across the IDE pair. This technique provides a spatial resolution up to the diffraction limit $(\approx 2 \mu \mathrm{m})$. In the left image the microchannel is outlined as it was not visible in the original captured image.

Upon characterization of the device's electrothermal behavior, we patterned an array of 16 uDEP IDEs along a single channel, and extended the enhanced and localized switch-like operation of the UDEP device to demonstrate the multiplexed actuation capability. Through a series of incubation and wash steps, $2.8 \mu \mathrm{m}$-diameter goat-anti-mouse-IgG covered beads were bound to mouse-IgG coated surface through specific interactions on each element of the array. We selectively detached immunobound beads from each element of the array, by applying voltage (turning uDEP on) at each IDE pair, one-by-one (Figure 5a). In addition to multiplexed protein analysis, our platform enhances the DEP capability in statistical characterization of the force spectrum of biological interactions, since the multiplexed format enables performing force spectroscopy on multiple devices independently (Figure 5b), with high dynamic range in a single channel; minimizing inter-experiment variations that originate from disparities in buffer conditions and assay preparation steps. We envision a plethora of possibilities for performing rapid and inexpensive multiplexed protein biomarker analysis using this localized electronic actuation method. 


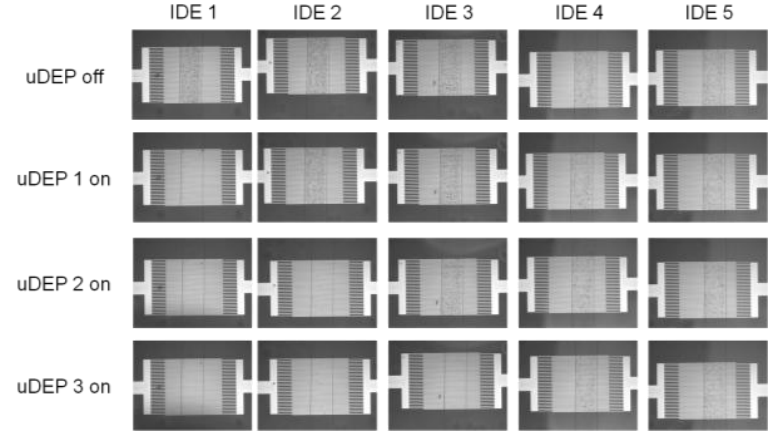

(a)

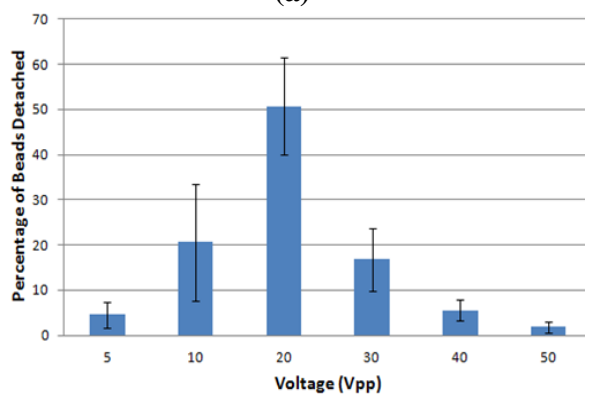

(b)
CONTACT

*S. Emaminejad, same@stanford.edu

Figure 5: (a) Five neighboring IDEs 1-5. Initially uDEP was off for all IDEs (row 1), and then uDEP was sequentially turned on by applying voltage at IDEs 1,2, and 3 (corresponding to rows 2,3, and 4). (b) Detachment percentage of protein-bound beads when ramping up the applied voltage at each of the IDE pairs one-byone. The results represent the collective detachment profile when actuating anti-IgG-IgG bound beads using our 16-plex platform. The array format allows performing force spectroscopy multiple times while ensuring operation under the same buffer conditions and assay preparation steps (DEP force is proportional to the square of the applied voltage).

\section{ACKNOWLEDGEMENTS}

This work was supported by the National Institutes of Health through grant PO1HG000205 and Natural Sciences and Engineering Research Council of Canada Scholarship through graduate tuition support. Fabrication of the devices was performed in the Stanford Nanofabrication Facility.

\section{REFERENCES}

[1] S. Emaminejad, M. Javanmmard, R.W. Dutton, and R.W. Davis, "Use of Negative Dielectrophoresis for Selective Elution of Protein-Bound Particles", Analytical Chemistry, 84 (3), pp 1432-1438 (2012).

[2] S. Emaminejad, M. Javanmmard, R.W. Dutton, and R.W. Davis, "Smart Surface for Elution of Protein-Protein Bound Particles: Nanonewton Dielectrophoretic Forces Using Atomic Layer Deposited Oxides", Analytical Chemistry, 84 (24), pp 10793-10801 (2012).

[3] M. Javanmmard, S. Emaminejad, C. Gupta, J. Provine, R.W. Davis, and R.T. Howe, "Depletion of Cells and Abundant Proteins from Biological Samples by Enhanced Dielectrophoresis", Sensors and Actuators B: Chemical, vol. 193, pp. 918-924 (2013).

[4] S. Emaminejad, M. Javanmmard, R.W. Dutton, and R.W. Davis, "Ultra Dielectrophoresis Using Atomic Layer Deposited Films: Electrothermal Analysis", ECS Transactions, 58 (10) 67-72 (2013) 